\title{
3D PSF Measurement for a Soft X-ray Microscope and Comparison to Theory
}

\author{
J. G. McNally, S. Rehbein, C. Pratsch, S. Werner, P. Guttmann and G. Schneider \\ Helmholtz-Zentrum Berlin, Albert Einstein Str. 1512489 Berlin Germany \\ james.mcnally@helmholtz-berlin.de
}

\begin{abstract}
The transmission X-ray microscope's 3D PSF exhibits an axial asymmetry that cannot be explained by an incoherent imaging model for either a lens or zone plate, with or without spherical aberration.

OCIS codes: (340.7460) X-ray microscopy; (180.6900) Three-dimensional microscopy; (110.2990) Image Formation Theory
\end{abstract}

\section{Introduction}

Transmission X-ray microscopy (TXM) using soft X-ray light ( $300-500 \mathrm{eV})$ is now an established and valuable technique for 3D ultrastructural examination of cells [1]. TXM's operate in bright-field, absorption-contrast mode, and most current instruments are based on the design of the Helmholtz-Zentrum Berlin (HZB) microscope, which uses an elliptically shaped single-bounce glass capillary as the condenser and a Fresnel zone plate as the objective [1]. The zone plate objectives have very small numerical apertures (NA 0.05). As a result, TXM's have relatively large depths of field, and so tomography based on a series of tilt angle images has been used to obtain 3D reconstructions of the specimen. However, as the resolution of the nanofabricated zone plates improves (now approaching $10 \mathrm{~nm}$ ), the depth of field of the TXM shrinks well below that of a typical cell's thickness, and as a result deconvolution becomes a more attractive option for 3D imaging by TXM.

A prerequisite for deconvolution is an accurate estimate of the microscope's point spread function (PSF). Although there have been some efforts to estimate TXM in-focus transfer functions [2], and in one case at several out-of-focus positions [3], there have been no direct measurements of a full 3D PSF for a TXM. Here we report such a measurement and compare the data to predictions of an imaging model for absorption-contrast, bright-field microscopy [4].

\section{Methods}

$60 \mathrm{~nm}$ gold nanoparticles were diluted in water. A small drop of this solution was added to an X-ray microscope grid, and then plunge frozen in liquid ethane. 3D images of isolated nanoparticles were obtained using the HZB TXM with a $25 \mathrm{~nm}$ zone plate and $\lambda=2.4 \mathrm{~nm}$ light. The pixel size in object space was $10 \mathrm{~nm}$, and the $z$ step size was $100 \mathrm{~nm}$. Theoretical calculations for the PSF were performed in Matlab. The Chebfun package was used for numerical integration of the highly oscillatory Fresnel integrals.

\section{Results and Discussion}

Our strategy to determine a soft X-ray microscope PSF was to start with a spherical object that can be easily measured with reasonable signal to noise. For this we chose a solid gold nanoparticle of $60 \mathrm{~nm}$ diameter. Although this is larger than the diffraction limit for the X-ray zone plate used $(25 \mathrm{~nm})$, our goal was to account for the 3D image of this nanoparticle, and then scale down the imaging model to sub-diffraction spheres.

The 3D nanoparticle images were corrected for temporal fluctuations in illumination intensity by normalizing the total intensity per focal plane to a constant. In addition, the data were slightly rotated computationally relative to the optical $(z)$ axis to correct for a small amount of lateral drift in the stage. Representative slices from the nanoparticle images (Fig. 1a,b) are displayed with an inverted intensity scale such that the brightest regions correspond to the highest amount of absorption. Note the asymmetrical axial intensity profile (Fig. 1b,c).

We compared these data to the imaging model used by Holmes and O'Connor [4] for deconvolution of images from a visible-light, bright-field microscope. They obtain an absorption PSF by calculating first the PSF of a point source emitting incoherent light. Then they essentially normalize this to one and finally subtract it from one. To compute an incoherent PSF for the TXM, we used Eq. 1 below to calculate the electric field for a point source emitting light that passes through a lens with focal length $f$ and with an aperture whose radius equals the zone plate radius $r_{z p}$ ( $K$ is the Fresnel constant and all other variables are defined in Fig. 1d): 


$$
E(\rho, \Delta z)=K \int_{0}^{r_{z p}} \rho^{\prime} d \rho^{\prime} \frac{\mathrm{e}^{i k r}}{r} \exp \left(-i k \frac{\rho^{\prime 2}}{2 f}\right) \exp \left(i k \frac{\rho^{\prime 2}}{2 R_{0}}\right) J_{0}\left(\frac{k \rho \rho^{\prime}}{R_{0}}\right)
$$

We used parameters corresponding to the design conditions for the $25 \mathrm{~nm}$ zone plate $\left(R_{f}=950 \mu \mathrm{m}, R_{0}=3.5 \mathrm{~m}, f=\right.$ $949.74 \mu \mathrm{m}, r_{z p}=45.33 \mu \mathrm{m}$ ). We find that the calculated PSF (Fig. 2a) fails to capture the axial asymmetry present in the measured data.

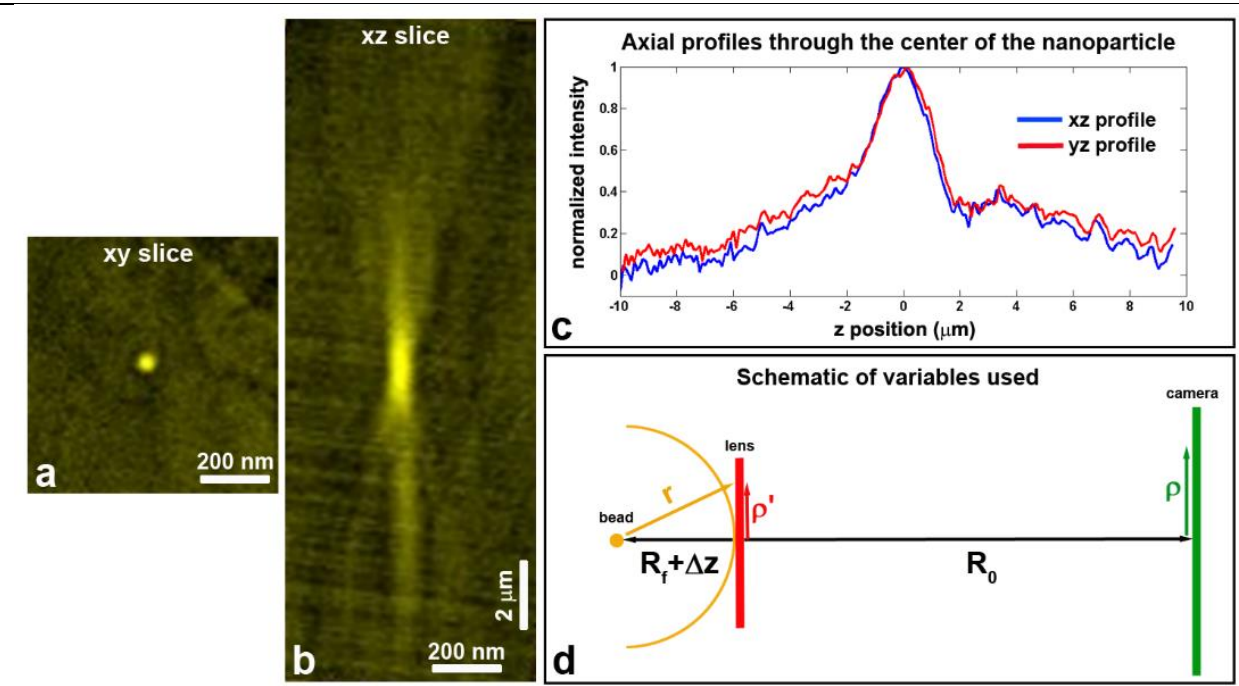

Figure 1. (a,b) xy and xz slices through the center of the $60 \mathrm{~nm}$ gold nanoparticle, contrast enhanced to illustrate the PSF tails. (c) The 3D nanoparticle image exhibits comparable axial asymmetry in xz and yz. (d) Schematic for the PSF theory.

One potential problem is that the calculated PSF is for a lens. Although it has been widely demonstrated that infocus PSFs of a zone plate and its equivalent lens agree, to our knowledge a direct 3D comparison of a zone plate PSF and the PSF of its equivalent lens has not been performed. Thus, we computed the 3D PSF of a zone plate by summing the diffraction patterns produced by a series of concentric clear zones corresponding to the zone plate rings. The electric field produced by the $N^{\text {th }}$ clear ring with outer and inner radii $r_{N, \text { outer }}$ and $r_{N \text {,inner }}$ is:

$$
E_{N}(\rho, \Delta z)=K \int_{r_{N, \text { inner }}}^{r_{N, \text { outer }}} \rho^{\prime} d \rho^{\prime} \frac{e^{i k r}}{r} e^{\left(i k \frac{\rho^{\prime 2}}{2 R_{0}}\right)} J_{0}\left(\frac{k \rho \rho^{\prime}}{R_{0}}\right)
$$

We obtained the 3D PSF for a zone plate by summing the $E_{N}$ in Eq. 2 for all of the clear zones. The specific parameters were for a Soret zone plate with 450 clear zones and a focal length of $949.74 \mu \mathrm{m}$, with the outer and inner radii given by $r_{N, \text { outer }}^{2}=2 N \lambda f$ and $r_{N, \text { inner }}^{2}=(2 N-1) \lambda f$. This approximates the geometry of the actual $25 \mathrm{~nm}$ zone plate used. The resultant 3D PSF was still symmetric and closely matched the 3D PSF calculated for the equivalent lens from Eq. 1 (Fig. 2a,b,g). These results demonstrate that imaging with a zone plate does not introduce axial asymmetry, and they also show that replacing a zone plate with an equivalent lens is acceptable for modeling the 3D imaging process in a TXM. These observations are consistent with the mathematical formulation of X-ray imaging in which each zone plate order gives rise to a term in the Fresnel integral that is equivalent to the quadratic phase term introduced by a lens [5].

Axial asymmetries in a microscope PSF can arise from aberrations in the optics [6]. One possible source of aberration on the HZB TXM is the camera location, which is at $R_{0}=1.9 \mathrm{~m}$ from the zone plate, even though the zone plate is designed for a camera at $R_{0}=3.5 \mathrm{~m}$. This can introduce spherical aberration [6], which will produce axially asymmetric PSFs. We therefore used Eq. 2 for a zone plate to calculate 3D PSF's for these two different camera locations. In addition, we also accounted for the specific radii of the actual zone plate used in the measurement, which were somewhat different than those of a Soret zone plate. We found that the 3D PSF's still exhibited axial symmetry (Fig. 2c,d,h), and specifically that the non-design location of the camera did not introduce any measurable spherical aberration into the microscope (Fig. 2d).

Finally, we wondered whether there might be some unknown source of spherical aberration present in the HZB TXM that could generate the measured asymmetric nanoparticle images. To test this possibility, we introduced different amounts of spherical aberration into the 3D PSF for the equivalent lens by multiplying the integrand in Eq. 1 by a fourth order term in the radial coordinate $\exp \left(i k W_{040}\left(\rho^{\prime} / r_{z p}\right)^{4}\right)$. Here $W_{040}$ is the number of waves of spherical aberration [7]. By introducing one wave of spherical aberration, we found that we could partially mimic 
the asymmetrical shape of the axial image of the gold nanoparticle, but only with a substantial contrast enhancement of the calculated incoherent PSF (Fig. 2e,f). A quantitative profile reveals that the axial tails of the measured nanoparticle image were significantly brighter than the axial tails in the PSF of the equivalent lens with one wavelength of spherical aberration (Fig. 2i). These results suggest that some hidden source of spherical aberration in the TXM cannot account for the measured nanoparticle images.
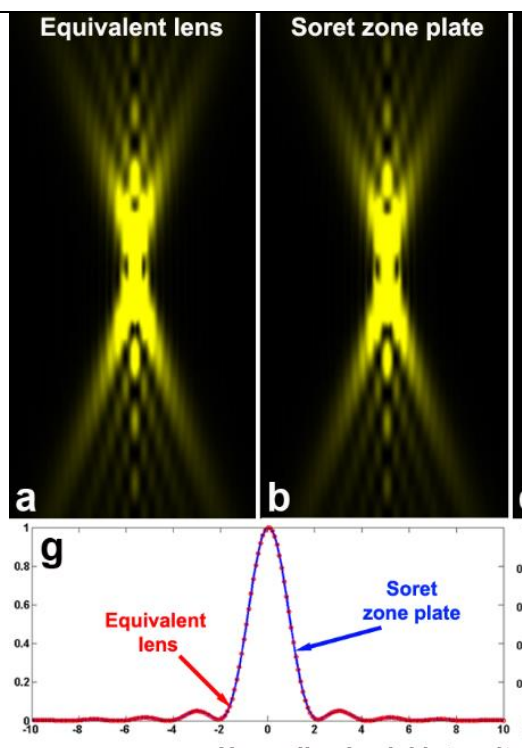

Normalized axial intensity
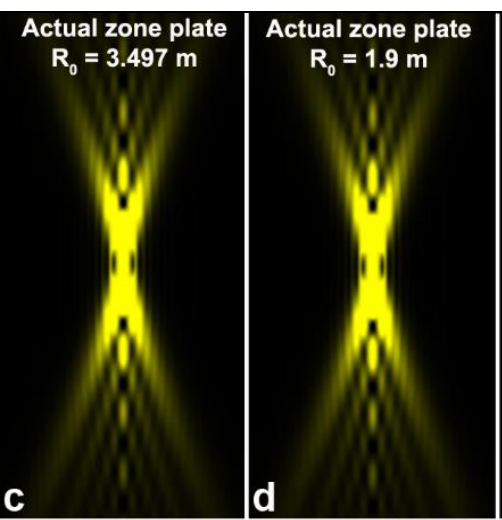

h
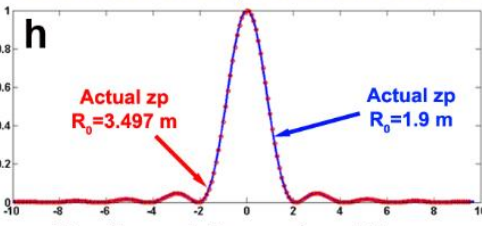
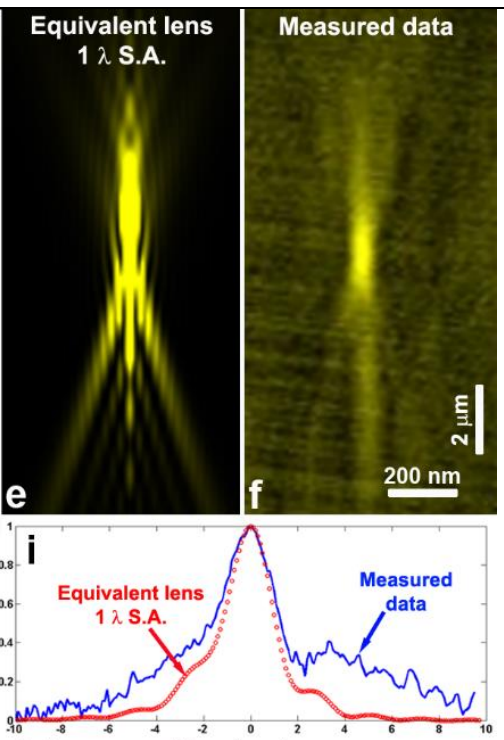

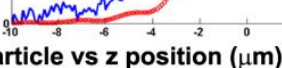

Figure 2. Axial profiles of computed PSFs displayed on an inverted intensity scale for: (a) an equivalent lens; (b) a Soret zone plate (zp); (c) the actual zone plate with design camera location; (d) the actual zone plate with non-design camera location; (e) an equivalent lens with one wave of spherical aberration (S.A.); (f) the measured data from the gold nanoparticle. All PSF images are contrast enhanced. The axial peak is defined as in-focus, and its $z$ position is then set to zero. Note the quantitative agreement between the PSFs for the equivalent thin lens and for various zone plates, even when used with different camera locations $(\mathrm{g}, \mathrm{h})$. Artificial introduction of one wave of spherical aberration cannot account quantitatively for the measured data (i).

In sum, our results show that an incoherent imaging model cannot accurately describe the measured gold nanoparticle images on the HZB TXM. This suggests that more sophisticated models that incorporate other properties of the microscope, such as its complex condenser design, effects of transmitted light and order sorting apertures and/or partial coherent imaging $[1,8]$ will be necessary for a proper theoretical explanation of the microscope's PSF. Development and experimental testing of such models will be critical for improved image restoration of soft X-ray microscope images.

\section{References}

[1] G. Schneider, P. Guttmann, S. Heim, S. Rehbein, F. Mueller, K. Nagashima, J. B. Heymann, W. G. Müller and J. G. McNally, "Threedimensional cellular ultrastructure resolved by X-ray microscopy,” Nat. Meth. 7, 985-987 (2010).

[2] S. Rehbein, P. Guttmann, S. Werner and G. Schneider, "Characterization of the resolving power and contrast transfer function of a transmission X-ray microscope with partially coherent illumination,” Opt. Express 20, 5830-5839 (2012).

[3] J. Otón, C. O. S. Sorzano, R. Marabini, E. Pereiro and J. M. Carazo, "Measurement of the modulation transfer function of an X-ray microscope based on multiple Fourier orders analysis of a Siemens star," Opt. Express 23, 9657-9572 (2015).

[4] T. J. Holmes and N. J. O'Connor, "Blind deconvolution of 3D transmitted light brightfield micrographs," J. Microsc. 200, 114-127 (2000).

[5] G. Schneider, “Cryo X-ray microscopy with high spatial resolution in amplitude and phase contrast," Ultramicrosc. 75, 85-104 (1998).

[6] S. F. Gibson and F. Lanni, "Experimental test of an analytical model of aberration in an oil-immersion objective lens used in threedimensional light microscopy," JOSA A 9, 154-166 (1992).

[7] J. C. Wyant and K. Creath, "Basic wavefront aberration theory for optical metrology" in Applied Optics and Optical Engineering, Vol XI (Academic Press, 1992), pp. 1-22.

[8] M. Selin, E. Folgelqvist, A. Holmberg, P. Guttmann, U. Vogt and H. M. Hertz, "3D simulation of the image formation in soft x-ray microscopes," Opt. Express 22, 30756-30768 (2014). 